\title{
Multi-View Face Detection Based on Improved Adaboost Algorithm
}

\author{
Han Zishuo ${ }^{1, a}$, Wang Chunping ${ }^{2, b}$ \\ 1,2Shijiazhuang Campus, Army Engineering University, Shijiazhuang Hebei, 050003, China \\ ashuo1986andy@126.com,bwchp17@139.com
}

Keywords: Face Detection; Adaboost Algorithm; Skin Color Detection

\begin{abstract}
Aiming at the problem of the traditional Adaboost algorithm with fast detection but low accuracy in multi-view face detection, a modified Adaboost algorithm for face detection was proposed in this paper.Firstly,in order to excluding the interference of most non-face regions, the whole image area is detected with skin color segmentation algorithm,then we use cascade structure classifier trained by Adaboost algorithm which addes the extended Haar features to classify the human face candidate regions, thereby the human face can be posited. Experimental results show that the algorithm of this paper can not only effectively improve the human face detection rate and detection speed, but also reduce the false detection rate of multi-view face, in short, the algorithm has a good practical value.
\end{abstract}

\section{Introduction}

Face detection[1] is the process of searching any one given image by using a certain strategy to sure whether it contains human faces, if it does then return the position, size and pose. Face detection is a key technology in face information processing which is involved in face recognition, expression recognition and gender recognition, and it is also the most representative special case in common target detection. Because of its great application value in the digital video processing, visual inspection, authentication, visual communication and other aspects, face detection has attracted the much attention of researchers.

For a long time, many scholars have done a lot in face detection, and also put forward many methods, but most of the methods have been troubled by detection accuracy and speed[2]. Until 2001 Adaboost algorithm is presented by Paul Viola and Michael Jones[3], the accuracy and speed of face detection is greatly improved and face detection really go into practical application. However, it needs exhaustive search for all possible detection windows which costs a long time and limits the application of this algorithm. Therefore, this paper proposes an improved Adaboost algorithm. Firstly, skin color segmentation algorithm is used to detect the image regions and eliminate most of the interference of non-face areas, and then the face regions are detected with Adaboost algorithm added the extended Haar features, thus achieving the purpose of detecting the information of human face quickly and accurately. 


\section{Improved Adaboost Algorithm}

Adaboost $^{[4]}$ is an iterative algorithm, the core idea is training different classifiers(weak classifiers) for the same training set, then put these weak classifiers together to form a stronger classifier (strong classifier).

Extended Haar Features and Calculation Method. Haar feature is a simple rectangle feature which is defined as the difference between the sum of the pixels within the black and the white rectangle ${ }^{[5]}$. Viola and Rainer Lienhart et $\mathrm{al}^{[6]}$ have made 14 kinds of Haar features which all have good symmetry, so the frontal face can be well detected, while the detection results of side face are unsatisfactory. For multi-view face detection, fully symmetric Haar features have been unable to perfectly describe sample characteristics. Yiqiang $\mathrm{Zhu}^{[7]}$ proposed four kinds Haar characteristics of asymmetrical structure in 2006, which is good for asymmetric face detection.

This paper used four types of Haar features, the structure shown in Fig1. There are three classes of symmetric Haar features (edge features, the line features and a central feature) and one class of asymmetric Haar features, shown in Fig1. Combining four Haar features can make a more complete description of the target.

1. Edge features
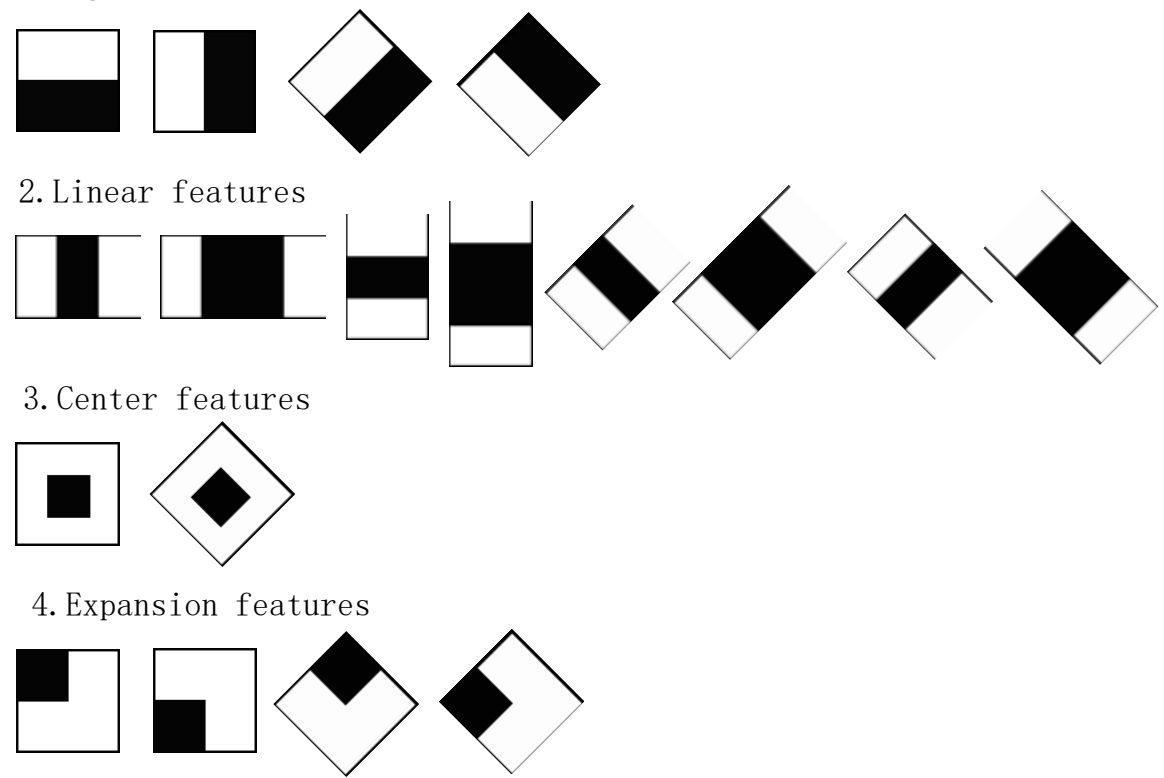

Fig1. Haar Feature Structures

Rectangle feature is defined as follows, feature $=\sum_{i \in(1 \ldots N)} \omega_{i} \operatorname{Re} c t \operatorname{Sum}\left(r_{i}\right)$.

In this formula, $\omega_{i}$ is the weight of rectangle $r_{i}$, RectSum $\left(r_{i}\right)$ is the sum of pixel values of all pixels in the rectangle $r_{i}$, $\mathrm{N}$ represents the number of the rectangles composed feature .

Integral Image. The integral image can quickly calculate the rectangle feature value. Let $I(x, y)$ present the arbitrary point of an image gray value, thus the integral image value of the point is defined as: 


$$
i i(x, y)=\sum_{x^{\prime} \leq x, y^{\prime} \leq y} I\left(x^{\prime}, y^{\prime}\right)
$$

For the integral image of any point on one image, only needs to sweep the original image once:

$$
\begin{aligned}
& s(x, y)=s(x, y-1)+i(x, y) \\
& i i(x, y)=i i(x-1, y)+s(x, y)
\end{aligned}
$$

In these formula, $s(x, y)$ is the ranks integral value of the location of point $(x, y)$, and $s(x,-1)=0, \quad i i(-1, y)=0$.

Adaboost Algorithm with Cascade Structure. Each stage of face detection classifier with cascade structure is trained by Adaboost algorithm. With Adaboost algorithm, the weak classifiers generated by Haar features superimpose into a strong classifier which has strong generalization ability. The specific training process of strong classifier is as follows:

(1) Given one training set $\left(x_{1}, y_{1}\right), \ldots,\left(x_{n}, y_{n}\right), x_{i}$ is the sample description and $y_{i} \in(0,1)$ is sample identification in this set, 0 and 1 represent face and non-face.

(2) Initialization: Set initial sample weight to be $\omega_{1, j}=\frac{1}{n}$.

(3) For $t=1,2, \ldots T$, loop executes the following steps:

(1)The normalized weight: $q_{t, i}=\omega_{t, i} / \sum_{j=1}^{n} \omega_{t, i}$.

(2)For each feature $f$, train one weak classifier $h(x, f, p, \theta)$, calculate the weighted error rate of all features of the weak classifiers : $\varepsilon_{f}=\sum_{i} q_{i}\left|h\left(x_{i}, f, p, \theta\right)-y_{i}\right|$.

(3) Select the best weak classifiers selected by the minimum error rate.

$$
\begin{aligned}
& \varepsilon_{t}=\min _{f, p, \theta} \sum_{i} q_{i}\left|h\left(x_{i}, f, p, \theta\right)-y_{i}\right|=\sum_{i} q_{i}\left|h\left(x_{i}, f_{t}, p_{t}, \theta_{t}\right)-y_{i}\right| \\
& h_{t}(x)=h\left(x, f_{t}, p_{t}, \theta_{t}\right)
\end{aligned}
$$

(4)According to this optimal weak classifiers, adjust the weights.

$$
\omega_{t+1, i}=\omega_{t, i} \beta_{t}^{1-e_{i}}, \beta_{t}=\frac{\varepsilon_{t}}{1-\varepsilon_{t}}
$$

In this, $e_{i}=0$ means that ${ }^{X_{i}}$ is correctly classified, $e_{i}=1$ means that ${ }_{i}$ is misclassified.

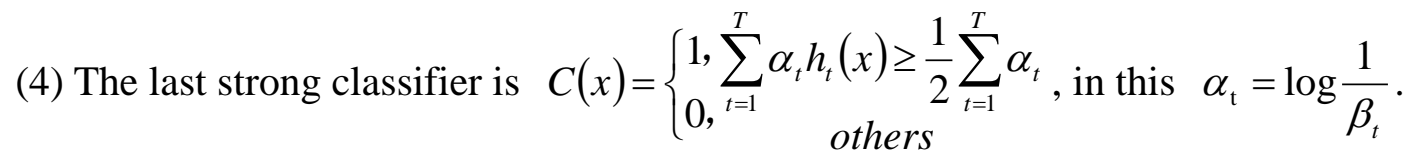


Face detection classifier with cascade structure ${ }^{[8]}$ is composed of multilevel strong classifiers. Each level strong classifier is trained by Adaboost algorithm. The structure of cascade classifier is shown in Fig2.

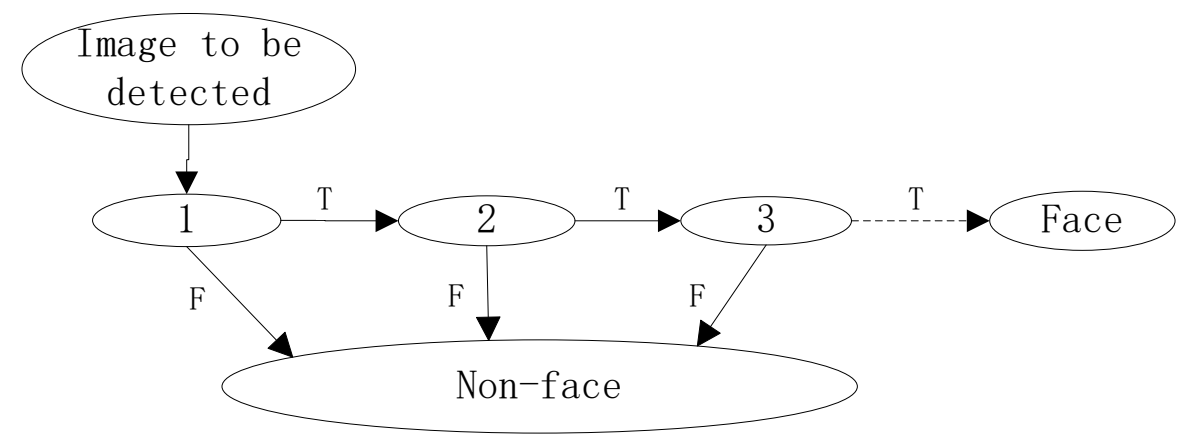

Fig2. Cascade Classifier Structure

Cascade classifier series several strong classifiers together, and every level of these classifiers is more complex and strict than next. The first few levels of classifier using fewer features have simple structure, so it can exclude a large number of non-face areas by a small amount of calculation. The later few levels of classifier using more features have relatively complex structure, so it can well separate face areas from similar backgrounds. Moreover, the first few levels of classifier have filtered most background areas out and leave only few areas which need to be filtered by next few levels of classifier, so the detection speed of Adaboost algorithm is sped up.

\section{The Improved Algorithm}

The main disadvantage of Adaboost algorithm which limits its practical application is that all areas of the image need exhaustive search which result in classifier training consumes too long time. In this paper, firstly, with the skin color detection we quickly eliminate most non-face areas, effectively reduce the face detection range, and then use the extended Adaboost algorithm with cascade structure to classify the face candidate areas, which has greatly improved the human face detection rate.

Light Compensation. Adaboost algorithm and skin detection are often subjected to the color deviation caused by imbalance lighted image. To counteract this color deviation existed in the image, the brightness of all pixels of entire image are arranged from high to low and take the top $5 \%$ of the pixels. If the number of these pixels is enough, their brightness will serve as "reference white" ${ }^{[9]}$.The $\mathrm{R}, \mathrm{G}, \mathrm{B}$ of the image are adjusted to the maximum value of 255 . Other pixel color values of entire image are also transformed by this adjustment scale.

Skin Color Detection. Because of the color clustering characteristic of skin color in $\mathrm{YC}_{\mathrm{b}} \mathrm{C}_{\mathrm{r}}$ color space, this paper chooses $\mathrm{YC}_{b} \mathrm{C}_{\mathrm{r}}$ color space as the statistical mapping space of skin distribution. In this $\mathrm{YC}_{b} \mathrm{C}_{\mathrm{r}}$ space, $\mathrm{Y}$ component represents the color brightness, $\mathrm{C}_{\mathrm{b}}$ and $\mathrm{C}_{\mathrm{r}}$ respectively represent the blue and red chromaticity, and the skin color on the $\mathrm{C}_{\mathrm{b}}$ and $\mathrm{C}_{\mathrm{r}}$ components are distributed in a relatively stable range: $80 \leq C_{b} \leq 135,136 \leq C_{r} \leq 177$. The $C_{b}$ and $C_{r}$ components found in this region are all considered as skin area which will enter the next round of Adaboost algorithm testing.

According to the geometric characteristics of color connected regions, such as the proportion of regional length, width, color, the non-face areas are removed significantly. The smaller Length and width rectangular skin areas are reserved, because the face feature extraction process can't provide 
sufficient detail for face detection. Skin detection result is shown in Fig3.
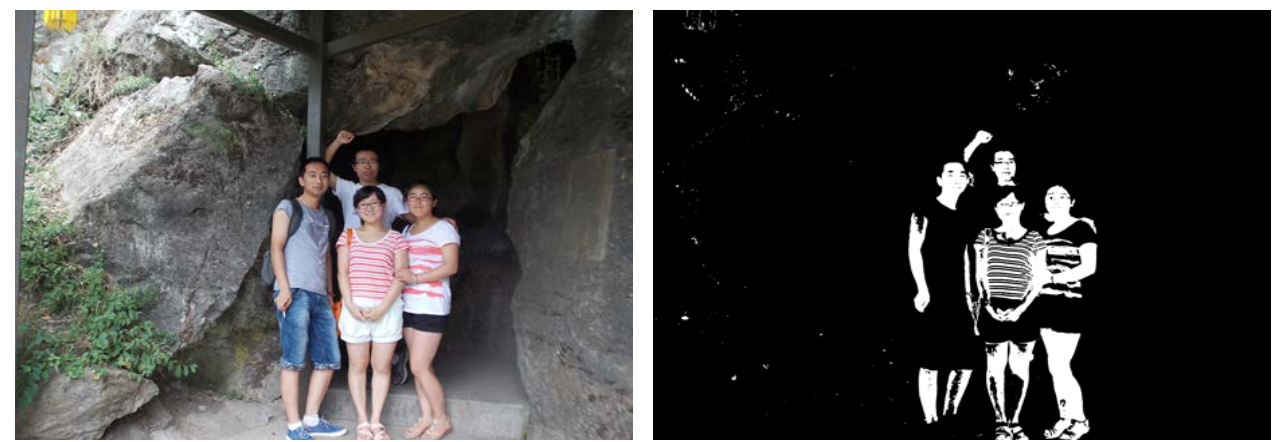

Fig3.Skin Detection Result

Algorithm Implementation Process. In order to reduce the influence of light on the image content, firstly, the illumination compensation is used to proceed the input sample image , and face detection and segmentation will complete the next phase of work, then most non-face areas are eliminated and the workload is reduced; secondly, eliminates the effects of noise on the skin color segmentation by etching, swelling and filtering the segmented areas, then according to statistical characteristics of the face areas, filters the candidate face region; finally use trained cascade Adaboost classifier to detect candidate areas, achieve accurate positioning of the human face, thereby improve the detection rate, reduce the false alarm rate. The algorithm process is shown in Fig 4.

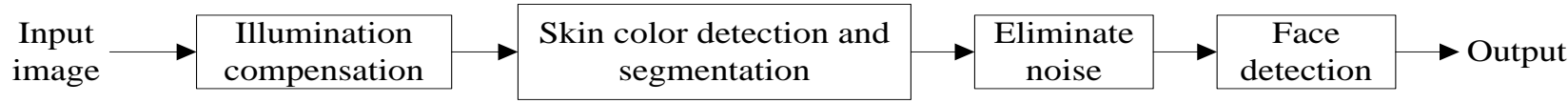

Fig4.Algorithm flowchart of this paper

\section{Experimental Results and Analysis}

The two sample sets are used in the simulation experiment, one is the front side sample set of the MIT + CMU, the next is self-built library sample set. 500 images are selected which facial expressions and details all have changes, for example, laughter or not, wear or do not wear glasses, front or side of the face, face size changes etc from the MIT + CMU. Then 200 images are selected from the self-built library sample set. Each image includes one or more than one face, most of them are different attitude and even small part of the face is obscured.

The test environment is that hardware system is the computer have Intel i5 2.3GHzCPU and 4G memory; the software are VC ++6.0 , OpenCV. Test results are shown in Table 1 . 
Table 1. The Results Contrast

\begin{tabular}{|c|c|c|c|c|}
\hline Algorithm used & $\begin{array}{l}\text { Training } \\
\text { Sample set }\end{array}$ & $\begin{array}{l}\text { Detection } \\
\text { time [s] }\end{array}$ & $\begin{array}{l}\text { Detection } \\
\text { rate [\%] }\end{array}$ & $\begin{array}{l}\text { False Detecting } \\
\text { rate [\%] }\end{array}$ \\
\hline Adaboost algorithm & \multirow{3}{*}{$\mathrm{M} \mathrm{IT}+\mathrm{CMU}$} & 63 & 84.6 & 7.8 \\
\hline The new algorithm of Baohu $\mathrm{Y}^{[10]}$ & & 48 & 93.4 & 4.5 \\
\hline The algorithm of this paper & & 39 & 94.6 & 3.1 \\
\hline Adaboost algorithm & \multirow{3}{*}{$\begin{array}{l}\text { Self-built } \\
\text { Sample set }\end{array}$} & 69 & 88.5 & 8.2 \\
\hline The new algorithm of Baohu Y & & 51 & 93.8 & 5.4 \\
\hline The algorithm of this paper & & 42 & 95.7 & 2.6 \\
\hline
\end{tabular}

As can be seen from the experimental data,multi-view classifier train by Adaboost algorithm have more false alarms and detection time is longer. The improved algorithm of this paper is better than Adaboost algorithm and the new algorithm of Baohu Yong in detection rate and speed. Fig 5 shows the test results of the application of this algorithm for face detection.
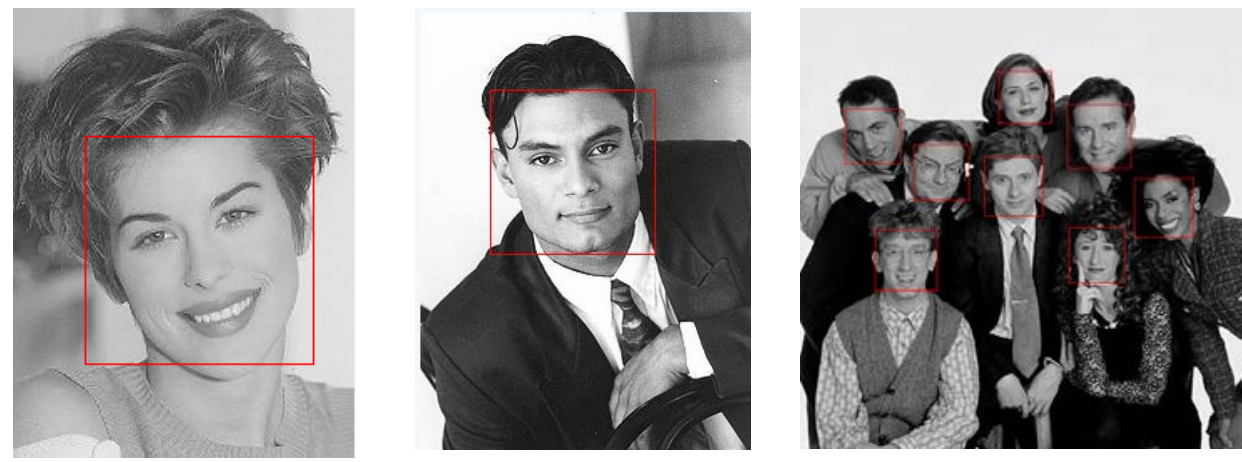

a. The Test Results of Sample Image frome M IT + CM U
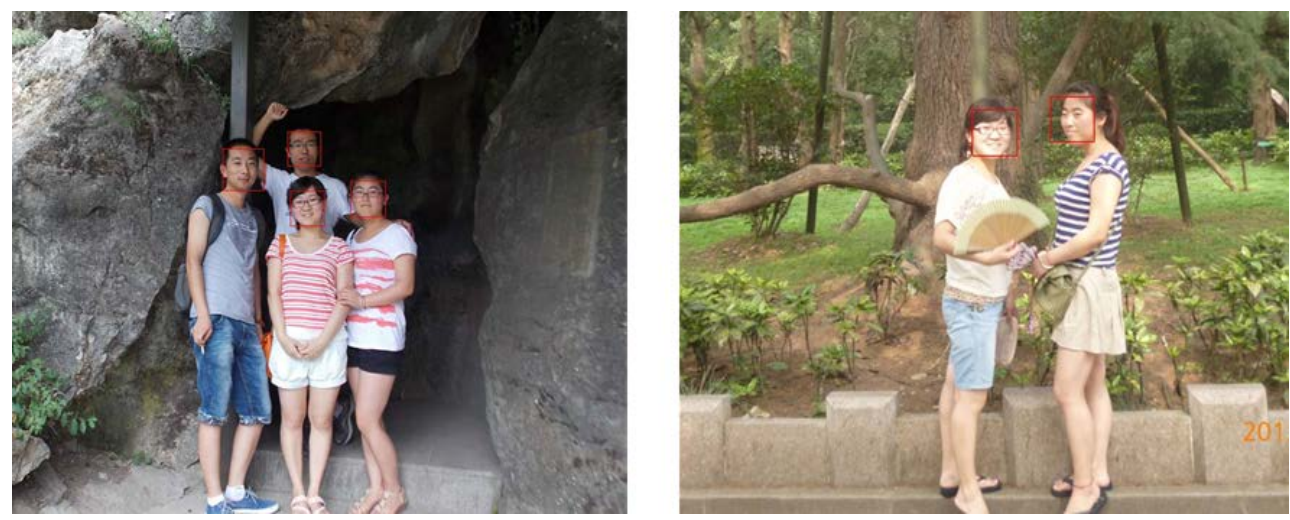

b. The Test Results of Sample Image frame Self-built Sample Set

Fig5. Some Experimental Results

\section{Conclusion}

Face detection is a complex pattern recognition problem. In this paper, the Adaboost algorithm is improved for twice by adding extended Haar features and skin color detection. Compared with other algorithms, the algorithm first detects the skin of samples, remove most of the non-face areas which effectively narrows the scope of face detection, and then uses cascade structure classifier trained by Adaboost algorithm which addes the extended Haar features to classify the human face 
candidate regions, thereby the human face can be posited. Experimental results show that the algorithm of this paper can not only effectively improve the human face detection rate and detection speed, but also reduce the false detection rate of multi-view face, in short, the algorithm has a good practical value.

\section{References}

[1] Luhong L, Haizhou A. Face detection research [J]. Chinese Journal of Computers, 2002, 25(5): 449-458.

[2] Rowley H A, Baluja S, Kanade T. Neural Network-based Face Detection[J].IEEE Trans. on Pattern Analysis and Machine Intelligence, 1998, 20(1): 23-28.

[3] P. Viola, M. Jones, Rapid object detection using a boosted cascade of simple features: Computer Visio and Pattern Recognition, 1 (2001), 511-518.

[4] Li K, Liping W. Face detection based on modified Adaboost algorithm[J]. Opto-Electronic Engineering, 2012, 39(1): 113-118.

[5] Y. Ju. Research of Feature Selection and Comparison in AdaBoost based Object Detection System . Journal of Computational Information Systems . 2013, 9(22): 8947-8954.

[6] Yiwei W. Muti-view Face Detection Based on Skin Color and AdaBoost Algorithm[D]. Beijing Jiaotong University thesis. 2011.

[7] Yiqiang Z. Real-time pedestrian detection system based on Adaboost algorithm[D]. Northwestern polytechnical university thesis, 2006.

[8] JONES M, VIOLA P. Fast multi-view face detection [EB/OL]. [2009-02-16]. http: //www.mer.l com /reports/docs/TR2003-96. Pdf

[9] Yang Jie, Lu W, Wa Bel A. Skin Color Modeling and Adaptation[A]. Proc. of the 3rd Asian Conference on Computer Vision[C]. London: Springer Verlag, 1998:687-694.

[10] Baohu Y, Lan L. Face Detection Based on Skin Color Segmentation and AdaBoost Arithmetic[J]. Science Technology and Engineering, 2013, 13(11): 3119-3225. 